Published in Human Interaction and Emerging Technologies. Proceedings of the 1st International Conference on Human Interaction and Emerging Technologies (IHIET 2019), August 22-24, 2019, Nice, France. Advances in Intelligent Systems and Computing, vol 1018. Ahram T., Taiar R., Colson S., Choplin A. (eds). Springer, Cham, 2019. ISBN:978-3-030-25628-9. https://doi.org/10.1007/978-3-030-25629-6_63

\title{
Using Dynamic Real-time Haptic Mediation in VR and AR Environments
}

\author{
Ahmed Farooq, Patrick Coe, Grigori Evreinov, Roope Raisamo \\ Tampere Unit of Human Computer Interaction (TAUCHI), Faculty of Information \\ Technology and Communication, Tampere University, Finland \\ \{Ahmed.Farooq, Patrick Coe, Grigori.Evreinov, Roope.Raisamo\}@TUNI.fi
}

\begin{abstract}
As VR, and AR become more popular forms of interaction with standalone and wearable computing systems, there is an inherent need to redefine the role of haptics in virtual environments. Complex virtual environments require more comprehensive tactile information. For this reason, primitive tactile signals currently being used in most commercial systems, need to give way to more precisely calibrated actuation, which is specifically designed for various applications. In this research we have extended the "Haptic Mediation" concept to test and improve haptic actuation for virtual interaction by developing a Dynamic Self-sensing and Actuation Architecture (DSAA) using Autonomous Haptic Devices (AHD). We have developed autonomous mobile versions of small haptic devices with embedded actuators, sensors, power sources as well as a radio communication channel (transceiver). These haptic devices can be attached to any part of the body to perform sensing and actuation. Our testing shows that this approach can improve users' overall experience in VR games and that AHDs can create reliable, perceivable actuation signals even when the user is actively moving around.
\end{abstract}

Keywords: Human-System Integration, Multimodal Interaction, Haptics.

\section{Introduction}

Our ability to learn and adapt, creates the basis of our interaction in new virtual environments. However, these are limits on how much the mechanics of a virtual system can vary, to still be considered usable reliable and similar to the physical space. When an external system utilizes commonly used real world interaction techniques (i.e. door knob being rotated clockwise or anticlockwise to open a door), the user of the system is easily able to transition into this interaction paradigm, even if the environment or its surroundings vary considerably. Current virtual environments are now pushing the boundaries of what is physical and what is virtual. These environments have more complex and enriching 3D worlds that are governed by intricate laws of interaction, perhaps even slightly different from the physical world (random spawning, teleporting etc.). These visually enhanced 3D environments should increase user immersion but in some case they do not. In fact, users identify a clear disconnect [14] between the $3 \mathrm{D}$ enhanced virtual and physical environments. This is because these virtual envi- 
ronments may have photo-realistic visual interfaces and 3D surround audio, but fundamentally come into conflict with our imagination and physical experiences due to the lack of meaningful haptic feedback. Thus, although users may be interacting with these environments directly (using their hands) or indirectly (using intermediate controller), the absence and immersive haptic feedback creates the fundamental gap.

Studies have shown that haptic feedback increases performance over visual only feedback in a wide range of tasks [1]. In fact, research by Cheng et al. [2] shows that by the addition of simple vibrotactile feedback it is possible to significantly improve task completion times in virtual environments. Similar research by Moehring and Froehlich [3] illustrates that addition of vibrotactile actuation signals with reference to grasping and manipulating virtual object can greatly increase system immersion. However, addition of haptic feedback does not always improve system interaction. As identified by Pawar and Steed [4], natural feedback cues are critical in creating immersive environment mechanics. Moreover, delays and inconsistent force feedback [5] parameters can negatively impact user perception. Furthermore, large heavy tethered wearable devices [6] can also reduce the immersive effect. Therefore, it is essential to provide context specific vibrotactile and kinesthetic actuation signals without using large heavy mechanical devices that need to be tethered to control and power sources for effective visualization and actuation [7].

\section{Dynamic Self-sensing and Actuation Architecture}

To achieve more complex and precise actuation signals it is important to calibrate and control not only the actuation signals but the actuation itself in real time. For this reason, we developed a self-encompassing actuation setup. Using the Haptic Mediation concept [7], we developed a Dynamic Self-sensing and Actuation Architecture (DSAA). This architecture consists of an actuator, a battery, driving circuitry, a wireless transceiver and an onboard 6-axisgyroscope. The onboard gyroscope provides real time actuation feedback, which is needed to dynamically adjust the actuation signals and to over-come environmental noise. While the transceiver and battery ensure that the system can provide tether-less actuation yet still be controllable, if needed.

To test the DSAA and its efficiency we created two Autonomous Haptic Devices (AHD). To keep the size as small as possible we utilize components that would be useful for mobile wearable devices or addons. We utilized an 8-ohm nominal Techtonic TEAX09C005-8 miniature voice coil actuator, which is optimized for electrodynamic transducer and has wide bandwidth and wide directivity. To drive the actuator, we used an Adafruit Feather M0 Bluefruit with onboard Bluetooth Low Energy (BTLE) and built in USB and battery charging. Driving signal from the M0 was amplified using an L298N high voltage, high current dual full-bridge driver. For dynamic actuation and motion sensing we used the UM6 r2 ultra-miniature orientation sensor as it has onboard gyro, accelerometer, magnetic sensor as well as a 32-bit ARM Cortex processor to compute sensor orientation up to 500 times per second. Powering the setup was a PKcell LP503035, 3.7v (1200mAh) Lithium polymer battery which was be recharged using the M0 Bluefruit main board. 


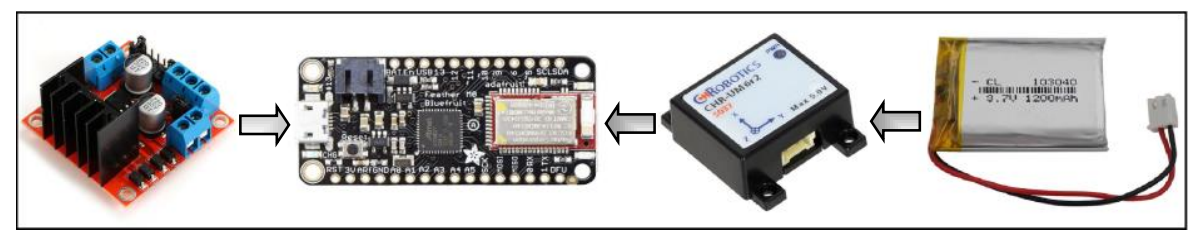

Fig. 1. (left to right) L298N signal amplifier, M0 Bluefruit Microcontroller with BTLE, UM6 r2 IMU and a LiPo battery.

\subsection{Dynamic Actuation Signals}

The two AHD devices use an onboard signal simulator which have preprogrammed haptic signals. These signals were designed to simulate a gunshot effect to the user. The signal was similar to the Immersion SDK actuation signal (Weapon_87 and Weapon 88 ), with an absolute value of $10 \mathrm{~Hz}$ applied for $175 \mathrm{~ms}$. Using this as a base signal, we developed 6 variations of the amended signals (S_F1 to S_F6) altering the frequency by a measure of $20 \%$ from the base signal. This meant that the application signal frequency varied from $4 \mathrm{~Hz}$ to $18 \mathrm{~Hz}$.

\subsection{VR System Integration}

The VR environment chosen for this research was a game, Overdrive. This is a popular shooting game that requires the player to avoid being struck by bullets and shoot the non-playing characters (NPCs). For the purpose of this research participants were instructed to not shoot at the NPCs but to simply avoid the bullets fired at them. This ensured that the participants did not have to use the Oculus controllers and were provided visual feedback in the game. The HMD used for VR interaction was the Oculus Rift ( $1^{\text {st }}$ generation), with no onboard audio feedback. The game was powered using an Alienware Laptop with a GTX 1070 mobile graphics card. The haptics feedback was generated using the audio feedback through the laptop system. The attachment was such that if the user was shot in the VR environment, the auditory feedback was used as a trigger to send a Bluetooth signal to the AHD devices (worn on the chest and back). Once the AHD devices were triggered, they either provided the 'Base application Signals' or a version of the 'Amended Actuation' depending on the experiment condition and user movements detected from the onboard motion sensor.

\section{User Study}

\subsection{Testing Methodology}

We conducted a basic user study with 24 student participants (10 male 14 female). The participants played 'OverDrive' using the Oculus Rift HMD without any controllers or audio feedback. Two AHDs were attached to the participants, one on the chest at the 'Sternum' and one at the back at on the 'Dorsum'. There were three separate conditions in which the participants were asked to play the game; sitting on a rotating chair; standing normally and jogging in a standing position. Each condition was com- 
pleted once the participants were shot 14 times, twice for every actuation signal. Once the participants perceived the haptic signal of being shot, they were asked to press a force sensitive resistive (FSR) button strapped (using a SEN-09375 ROHS sensor) to the palm of their dominant hand. Using this information researchers identified the response time and pressure \& force of the response of the participants, to each feedback signal in the three conditions like Kim et al., [8]. After each condition the participants were asked to specify how many times they were shot in the game. They were also asked to rate the 7 signals according to their perceptual force or strength and how pleasurable each signal was within the specific condition.

\subsection{Results and Discussion}

If we look at the time it took the users to press the FSR button on their palm (Fig. 3), we see that there is a clear trend for condition $\mathrm{C}$ between $\mathrm{S} 1, \mathrm{~S} 2, \mathrm{~S} 3$ and SB. As expected, lower frequency actuation signals took longer to be recognized by the users in condition C. Moreover, we see the same trend with amount of force by which the participants press the button, where lower frequency signals were followed by greater force presses of the FSR button, however, this trend evens out for the higher frequency actuation signals for all three conditions. Results also show that participants' response times did not vary through the seven actuation signals and the remaining two conditions (A \& B). This would suggest that the participants' response times and force applied to the button were not affected by the variations in frequency between the tested actuation signals, either positively or negatively, but by the jogging condition $(\mathrm{C})$.

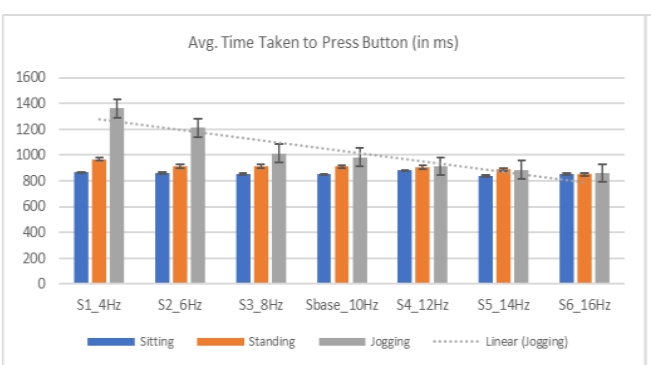

Fig. 3. Time taken to press the FSR button for all 3 conditions.

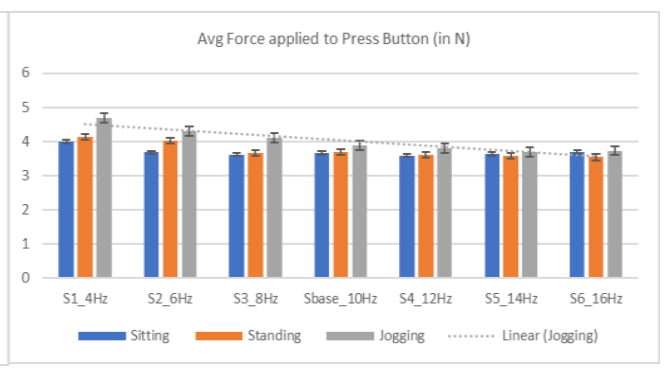

Fig. 4. Average force applied to the FSR button for all 3 conditions.

More interestingly when comparing the results of the recorded errors across the three conditions and seven actuation signals (Fig. 5), we see that the errors were much higher for condition $\mathrm{C}$ overall and specifically for signals with lower frequencies. This meant that the users either did not sense the applied signal or the signal was so weak that they did not identify it w.r.t the 'shooting event' in the VR environment. Moreover, if we compare the recorded errors with the participants perceived error rate, we see that on average the participants felt they performed more poorly than recorded. In fact, this can be seen throughout the results of condition $\mathrm{C}$, which points to the fact that participants were unsure of the signal while jogging and felt the haptic feedback was unreliable. 


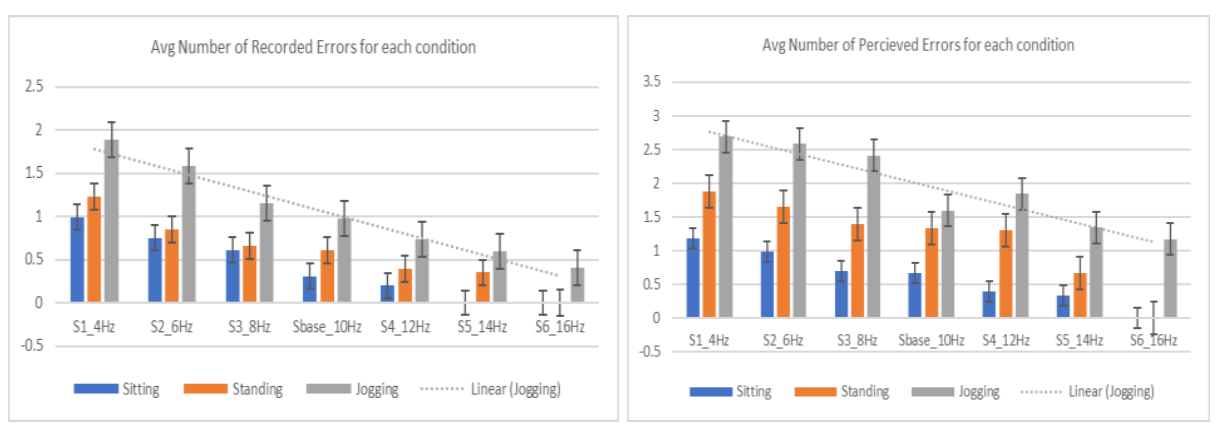

Fig. 5. Average number of recorded errors (left) compared to perceived errors (right) while pressing the FSR button for all 3 conditions.

Looking at the perceived sensitivity of the actuation signals over the three condition, we can again see from the result (Fig. 6) that lower frequency signals were felt less sensibly as a whole, specifically in condition C. On the other hand, pleasantness results suggest that user perceived the lower frequency signals to be felt more pleasant yet less sensible in different conditions. This trend is visible for all three conditions, however the variations for condition $\mathrm{A}$ and $\mathrm{B}$ is greater than for condition $\mathrm{C}$ for the same signals. This would mean that once the applied signal was sensible, the users preferred the lower frequency feedbacks over the higher frequency signal.

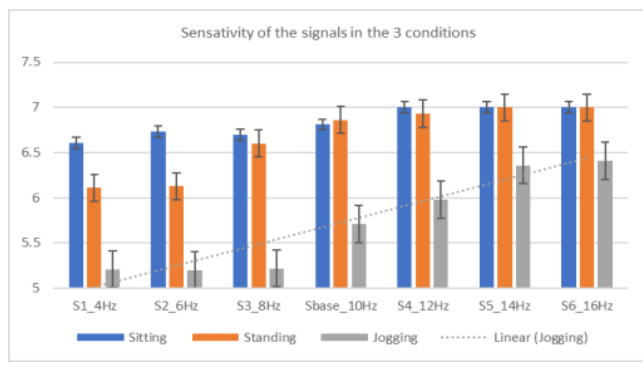

Fig. 6. Average Sensitivity rating for each applied signal over the 3 conditions.

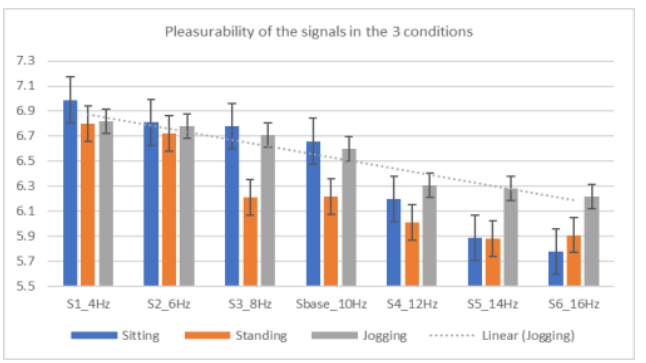

Fig. 7. Average Pleasantness rating for each applied signal over the 3 conditions.

\section{Conclusions}

In this research we have extended the dynamic haptic mediation technique to test and improve haptic feedback for virtual interaction by developing a Dynamic Self-sensing and Actuation Architecture (DSAA) using Autonomous Haptic Devices (AHD). Instead of using traditional techniques of having a single actuation signal for a VR based interaction event the AHDs can provide a larger scope of actuation signals depending on the environmental noise or movement of the user. To test the AHDs, we conducted a user study with 24 participants who played "OverDrive", a VR game using the Oculus Rift headset. Once the AHDs recognized that the participants were moving around the frequency of haptic feedback provided to the participants was 
adjusted according to their movements. Results demonstrate that dynamic haptic mediation improved the users' overall experience in the VR game and created a larger scope of haptic actuation rather than what is possible using current techniques. AHDs enhanced the actuation signal and increased the area of sensibility when the participants were actively moving around. Moreover, the variation of the signal did not create any adverse effects and ensured that when needed, the users could be provided the most pleasant actuation signal as possible. Similarly, in haptically noisy environments, the AHDs can provide a slightly altered signal to increase perceptual strength and reliability of the haptic feedback. Furthermore, this technique can filter out fringe frequencies generated by environmental vibration-noise (movement) and reduce the audio-component of the attached actuators. In our future research, we plan to explore how it may be possible to combine multiple AHDs over a range of actuation signals within a complex haptic stimulus yet keeping the signal perceptual integrity [7].

\section{Acknowledgement}

The research was supported Finnish Culture Foundation's Post-Doctoral mobility grant awarded to the PI and was also partially funded by Business Finland (MIVI Project, decision 8004/31/2018), and was carried out in collaboration with the Haptic Interface Research Lab (HIRL) at Purdue University, Indiana.

\section{References}

1. Burke, J. L., Prewett, M. S., Gray, A. A., Yang, L., Stilson, F. R. B., Coovert, M. D., Elliot, L. R., and Redden, E. (2006). Comparing the effects of visual-auditory and visual-tactile feedback on user performance: a meta-analysis. In Proceedings of the 8th international conference on Multimodal interfaces. ACM, 108-117.

2. Cheng, Li-Te, Kazman, R., and Robinson, J. (1997). Vibrotactile feedback in delicate virtual reality operations. In Proceedings of the fourth ACM international conference on multimedia. ACM, 243-251.

3. Moehring, M., and Froehlich, B. (2011). Effective manipulation of virtual objects within arm's reach. In 2011 IEEE Virtual Reality Conference. 131-138. https://doi.org/10.1109/VR.2011.5759451

4. Pawar, V. M., and Steed, A. (2009). Evaluating the in\$uence of haptic force-feedback on 3D selection tasks using natural egocentric gestures. In Virtual Reality Conference, 2009. VR 2009. IEEE. IEEE, 11-18.

5. Berg, D. V. D., Glans, R., Koning, D. D., Kuipers, F. A., Lugtenburg, J., Polachan, K., Venkata, P. T., Singh, C., Turkovic, B., Wijk, B. V. (2017). Challenges in Haptic Communications Over the Tactile Internet", Access IEEE, vol. 5, pp. 23502-23518

6. Blake, J., and Gurocak, H. B. (2009). Haptic Glove with MR Brakes for Virtual Reality. In IEEE/ASME Journal on Transactions on Mechatronics, Vol: 14, Issue: 5, Oct. 2009. DOI: 10.1109/TMECH.2008.2010934

7. Farooq, A. (2017). Developing technologies to provide haptic feedback for surface based interaction in mobile devices, PhD Thesis, University of Tampere, Faculty of Communication Sciences. http://tampub.uta.fi/handle/10024/102318.

8. Kim, J. H., Aulck, L., Bartha, M. C., Harper, C. A., Johnson, P. W. (2014). Differences in typing forces, muscle activity, comfort, and typing performance among virtual, notebook, and desktop keyboards. Applied ergonomics 45, 6 (2014), pp. 1406-1413. 